\title{
Lipidomic Analysis of Serum from Horses with Strongyle Infection
}

Jenna Lamb ${ }^{1}$, Gayle D. Hallowell ${ }^{1}$, Patricia Harris ${ }^{2 *}$, Xing-Quan Zhu ${ }^{3}$ and Hany M. Elsheikha ${ }^{1 *}$

${ }^{1}$ Division of Veterinary Medicine, School of Veterinary Medicine and Science, Faculty of Medicine and Health Sciences, University of Nottingham, Sutton Bonington Campus, Leicestershire, United Kingdom

${ }^{2}$ WALTHAM Centre for Pet Nutrition, Waltham-on-the-Wolds, Melton Mowbray Division of Veterinary Medicine, School of Veterinary Medicine and Science, Faculty of Medicine and Health Sciences, University of Nottingham, Sutton Bonington Campus, Leicestershire, LE14 4RT, United Kingdom.

${ }^{3}$ State Key Laboratory of Veterinary Etiological Biology, Key Laboratory of Veterinary Parasitology of Gansu Province, Lanzhou Veterinary Research Institute, Chinese

Academy of Agricultural Sciences, China

\begin{abstract}
The development of techniques capable of accurate diagnosis of strongyle infections is at the forefront of research in equine parasitology. The aim of this study was to evaluate the potential, for using lipidomics in the diagnosis of strongyle infection. Blood and fecal samples were collected from 30 horses. Fecal egg count (FEC) results were used to select the serum samples from six uninfected horses (negative controls) and from the five horses with the highest burdens. The lipid portion of serum samples was extracted and analyzed using High Performance Liquid Chromatography-Mass Spectroscopy. The concentrations of 66 lipid metabolites differed between infected and uninfected horses $(p<0.025)$. Database comparison of mass $/$ charge $(\mathrm{m} / \mathrm{z})$ ratios and retention times were used to tentatively identify 16 of these metabolites. The roles of these metabolites and reasons for the observable changes were discussed. These results demonstrate the potential for the use of high resolution lipidomic analysis, for the development of a diagnostic technique capable of detecting, and perhaps stratifying, equine strongyle infection.
\end{abstract}

Keywords: Strongyle; Biomarker; Metabolomics; Lipid profile; Lipidomics

\section{Introduction}

The family Strongylidae, which includes large strongyles (Strongylinae) and small strongyles (Cyathostominae), includes some of the most common and pathogenic parasites of horses. There are about 60 equine strongyle species [1], all capable of causing clinical disease termed strongylosis. Most of the species are so-called small strongyles or cyathostomes, for which infective third stage larvae migrate into the mucosa/submucosa of the large intestine. The large strongyle (Strongylus spp i.e. S. vulgaris) infective third stage larvae migration is within blood vessels. Strongylosis has a huge impact on the welfare of infected horses globally, with clinical effects ranging from mild depression and lethargy to profuse diarrhea, colic and a fatal wasting syndrome.

Diagnosis of strongyle infection in practice has been traditionally based on fecal analysis, with microscopic identification of eggs and parasite larvae. However, besides being labour-intensive and suffering from low diagnostic sensitivities, these techniques can't detect prepatent infection. This is a major limitation in some strongyle infections such as Strongylus vulgaris and cyathostomins, where most of the damage is attributed tolarval stages rather than adult worms. Theincreasing demand for accurate and reliable diagnostic tools has lead to the development of more sensitive and specific molecular diagnostic techniques, with the aim of eventually replacing the traditional techniques. New molecular techniques include a PCR-ELISA to amplify cyathostomins ribosomal intergenic spacer (IGS) region from fecal samples [2]; a reverse line blot assay that targets the IGS region of 13 species of cyathostomins and all 3 species of Strongylus [3]; a fluorescence-based real-time TaqMan PCR assay, to detect and semi-quantificate the DNA from Strongylus vulgaris in fecal samples [4]. Despite their promising potential, none of these molecular tests is capable of detecting all important parasites of horses in one analysis. Further research and development is required, before a fully quantitative diagnostic test can become commercially available.

Lipidomics have been used to study and understand the biological mechanisms of parasites, often with the aim of developing more specific antiparasitic treatments $[5,6]$. However, the effect of strongyle parasitism per se on the equid host's lipid profile has not been documented. This study aims to evaluate the use of metabolomics, to determine differences in the lipid profile of sera from strongyle infected versus non-infected horses. This will allow appraisal of this technique's potential for future development of a diagnostic test.

\section{Materials and methods}

\section{Sample collection}

Samples of blood and feces were taken from horses being euthanased, at an equine abattoir, for purposes other than research. These horses had originated from different owners and therefore, had been kept under different management practices, with an unknown history with respect to their anthlemintic treatment. Although a formal BCS evaluation was not undertaken, animals that were obviously thin or very fat were excluded from the study (i.e., samples were collected from animals of a similar BCS). Thirty sets of samples were collected. A single blood sample was collected immediately post euthanasia from the jugular and carotid veins of each horse. Transport and storage in $50 \mathrm{ml}$ falcon tubes in the absence of anticoagulants allowed blood to clot, in preparation for serum collection. On return to the laboratory, samples were stored in an upright position at about $4^{\circ} \mathrm{C}$ in a cold room.

*Corresponding author: Hany M. Elsheikha, Division of Veterinary Medicine, School of Veterinary Medicine and Science, Faculty of Medicine and Health Sciences, University of Nottingham, Sutton Bonington Campus, Leicestershire, United Kingdom, Tel. 00440115-951-6445; Fax: 0044-0115-951-6440; E-mail: Hany.Elsheikha@nottingham.ac.uk Patricia Harris, WALTHAM Centre for Pet Nutrition, Waltham-on-the-Wolds, Melton Mowbray Division of Veterinary Medicine, School of Veterinary Medicine and Science, Faculty of Medicine and Health Sciences, University of Nottingham, Sutton Bonington Campus, Leicestershire, LE14 4RT, United Kingdom.

Received August 20, 2012; Accepted September 17, 2012; Published September 20, 2012

Citation: Lamb J, Hallowell GD, Harris P, Zhu XQ, Elsheikha HM (2012) Lipidomic Analysis of Serum from Horses with Strongyle Infection. J Vet Sci Technol 3:122 doi:10.4172/2157-7579.1000122

Copyright: (C) 2012 Lamb J, et al. This is an open-access article distributed unde the terms of the Creative Commons Attribution License, which permits unrestricted use, distribution, and reproduction in any medium, provided the original author and source are credited. 
Samples from several fecal balls were collected from the rectum of each horse within $10 \mathrm{~min}$ of the blood sampling. These were immediately put into $50 \mathrm{ml}$ sealed plastic containers, that were numbered to match with each horse's corresponding blood sample. Fecal samples were also stored at $4^{\circ} \mathrm{C}$, to further minimize chances of egg development [7].

\section{Fecal egg count}

McMaster technique was used to detect and quantify strongyle eggs per gram (epg) in fecal samples as described [8]. Each sample was analyzed on three separate occasions using identical procedures and a mean egg count, \pm the standard deviation (SD) for each horse was calculated, to be used for analysis.

\section{Lipidomic analysis}

Serum Separation and Selection: All serum was removed from the surface of clotted blood samples and transferred to $1 \mathrm{ml}$ sterile eppendorf tubes, using a pipette. Tubes were left overnight in the cold room to allow sedimentation of any remaining blood cell contamination. Serum was again pipetted off, transferred to clean eppendorfs and frozen at $-20^{\circ} \mathrm{C}$. Once all fecal samples had been analyzed to assess the worm burden of each horse, serum samples from six horses with negative FEC and from the five horses with the highest burdens (> 500epg) were selected for analysis. Selection was based primarily on FEC results. Before analysis, sera from the control horses with negative FEC were pooled to produce one control sera sample. Samples were packaged appropriately and transported on ice to the Centre for Analytical Bioscience (CAB), University of Nottingham, where they were kept at $-80^{\circ} \mathrm{C}$.

Lipid Extraction: Lipids were extracted from the serum using a chloroform: methanol liquid-liquid extraction method based on the technique described [9]. This method separated lipids from all nonlipid elements, including any lysed red blood cells. The lipid containing layer was removed and mixed with an equal volume of isopropanol, prior to analysis. $10 \mu \mathrm{l}$ of lipid extract from each sample was then used for analysis. Quality controls were prepared using equivalent volumes of plasma from the $\mathrm{CAB}$ plasma library.

Liquid Chromatography: Liquid chromatography (LC) was used to separate different components of the extracted lipid samples. A pump causes the mobile phase (comprised of solvent and analytes) to move through the stationary phase(s), within the column used. The time taken for the sample to pass down the column and be eluted is defined as the retention time (RT). RT varies between analytes and can therefore be used for identification purposes. It is dependent upon the strength of sample interaction with the stationary phase(s), the ratio and composition of solvents used, and the flow rate of the mobile phase. An Accela High Performance Liquid Chromatography (HPLC) system was used to introduce samples onto a Thermosil Gold C18 3mm x 2.1 $\mathrm{mm}$ column (1.9mm particle size). Ten microlitres were injected on each occasion. Samples were eluted using a 5 minute water/acetonitrile/ isopropanol gradient, modified with $10 \mathrm{mM}$ ammonium acetate at $\mathrm{pH}$ 6.8 .

Mass Spectroscopy: The resulting eluent from the LC was ionised using an electrospray mode, in which the eluent is sprayed into a chamber at atmospheric pressure. A strong electric field causes further dissociation of analyte molecules, while a heated drying gas causes solvent in the droplets to evaporate. As droplets reduce in size, the internal charge concentration increases. Gaseous ions are ejected, once repulsive forces between ions with like charges exceed cohesive forces holding ions together. Gas ions enter the Exactive mass spectrometer. Samples and quality controls were injected 3 and 6 times respectively.
Settings allowed ions with $\mathrm{m} / \mathrm{z}$ ratios between 100 and 1500 to be detected, with the spectrometer being calibrated to within $1 \mathrm{mmu}$ and $2 \mathrm{mmu}$, respectively. The ratio of the concentration of these metabolites in uninfected samples to infected samples was calculated and presented as a decimal. Ratios less than 1 indicate an increase of the relevant metabolite in horses with strongyle infection, compared to those that were uninfected. A ratio greater than 1 is indicative of a reduction in metabolite level in infected horses.

Data analysis: Statistical differences between the infected and uninfected sample groups were highlighted using Sieve 1.2 software. Results were then filtered using Microsoft Excel to produce a final list of ions that showed statistical differences, defined as $\mathrm{p}<0.025$ (calculated using Student's T test) and strong MS signals (peak area $>5000$ ). Databases, including the Human Metabolome database and the laboratory's own accurate mass conversion of lipid maps, were screened for matching masses.

\section{Results}

\section{Fecal analysis}

Of the 30 horses whose fecal samples were analyzed, eight animals had consistently negative FEC results. 22 of 30 horses had strongyle burdens which ranged from barely detectable $(<50 \mathrm{epg})$ to heavy (approx $1200 \mathrm{epg}$ ) showing a high prevalence of infection $(73.3 \%)$ Other than eggs of ascarid nematodes, which were also seen in fecal samples from two horses, no parasitic elements other than strongyle eggs were identified. Blood samples from the five horses with the highest burden $(602 \pm 6.7,825 \pm 18.7,1005 \pm 119.5,1140 \pm 164.9$ and $1200 \pm 281.3 \mathrm{epg}$ ) were selected for serum analysis, and blood samples from six horses with negative FEC results were pooled and used as the negative control. This provided an estimation of normal metabolite concentrations in the lipid profile of equine serum.

\section{Serum analysis}

The concentrations of 66 metabolites were significantly different between infected horses and the pooled control sample $(p<0.025)$ Tentative identification of 16 metabolites was achieved based on their $\mathrm{LC}$ retention times and MS $\mathrm{m} / \mathrm{z}$ values (Table 1 ). The identifications could be grouped into cholesterol esters (including cholesteryl acetate, cholesteryl nitrolineate), phosphoglycerides (phosphoethanolamines, phosphocholines, diacyl-phosphoglycerides), triglycerides and sphingolipids (sphingomyelin, ceramide).

\section{Discussion}

The present study was conducted to determine, if there are detectable differences in the lipid metabolites in the serum of horses infected with strongyles, when compared to uninfected horses. This study found that the concentrations of 66 lipid metabolites were apparently changed when infection was present $(\mathrm{p}<0.025)$. The present study was also able to tentatively identify 16 of the 66 metabolites that differed between infected and uninfected horses. Full and specific identification of the lipid profile would require the existence of positive control standards, which were not available at this time. The 16 tentatively identified metabolites were grouped according to lipid class into sphingolipids, triglycerides, cholesterol esters and phosphoglycerides.

A large proportion of sphingolipids are found in cell membranes. Sphingolipid metabolism is involved in the regulation of cell growth, differentiation and apoptosis. Sphingolipids also have key roles in cell signaling [10]. Administration of exogenous sphingolipids has been 


\begin{tabular}{|l|l|l|l|}
\hline $\begin{array}{l}\text { Mass/charge } \\
\text { ratio } \mathbf{( m / z )}\end{array}$ & $\begin{array}{l}\text { Retention } \\
\text { time }(\mathbf{m i n})\end{array}$ & $\begin{array}{l}\text { Ratio control/ } \\
\text { disease }\end{array}$ & Potential IDs \\
\hline 429.3735 & 2.45 & 2.25 & Cholesteryl acetate \\
\hline 700.5295 & 2.66 & 0.57 & Phosphoethanolamine \\
\hline 716.5606 & 2.67 & 0.66 & Phosphocholine/cholesteryl nitrolineate \\
\hline 700.5306 & 2.81 & 0.64 & Phosphoethanolamine \\
\hline 814.6403 & 3.06 & 0.34 & Phosphoethanolamine/phosphocholine \\
\hline 790.6339 & 3.07 & 0.73 & Phosphoethanolamine/phosphocholine \\
\hline 816.5747 & 3.12 & 1.31 & Diacyl-phosphoglyceride \\
\hline 790.6228 & 3.16 & 0.57 & Diacyl-glycerophosphocholine \\
\hline 814.6294 & 3.18 & 0.32 & Diacyl-glycerophosphocholine \\
\hline 672.6282 & 3.21 & 0.65 & Ceramide \\
\hline 814.6918 & 3.25 & 2.20 & Ceramide/phosphocholine \\
\hline 836.6715 & 3.25 & 2.72 & Sphingomyelin \\
\hline 925.8151 & 3.40 & 1.95 & Triglyceride \\
\hline 799.6790 & 3.41 & 1.56 & Triglyceride \\
\hline 800.6835 & 3.41 & 1.98 & $\begin{array}{l}\text { Phosphoethanolamine/ } \\
\text { phosphocholine }\end{array}$ \\
\hline 669.5591 & 3.48 & 0.37 & Cholesteryl ester \\
\hline
\end{tabular}

Table 1: 16 identified metabolites found to have significant difference in levels between infected and uninfected horses.

shown to reduce Loxosceles gaucho spider venom spread within the host [11]. Although, the toxin involved in that study is not linked to strongyle infection, the results suggest that sphingolipids might have an effect on the establishment of parasite infections within the host. As the present study found a general reduction of sphingolipid concentrations in infected horses, this could mean that those animals which are not able to mount an adequate response including an increase in sphingolipids, may be those that are at higher risk of a large stronglye burden. Alternatively, as sphingolipids may be involved in the induction of an inflammatory reaction via the eicosanoid pathway [12], this could indicate that strongyles have developed a mechanism to reduce sphingolipid concentrations. This, in turn, potentially could reduce the impact of the parasitic burden on the host's health by minimizing inflammation, and by extending host survival, the parasites can persist and reproduce within the host for a longer period of time.

Increased serum triglyceride concentrations have been associated with the presence of systemic diseases including parasitism in miniature horses and donkeys [13]. This finding was associated with rapid mobilization of triglycerides from peripheral adipose tissue, triggered by the negative energy balance caused by the disease. However, reduced triglyceride concentrations, as identified in the present study, have been found in strongyle infected donkeys [13] and in horses suffering from chronic starvation, prior to lipid mobilization [14]. Parasitic damage to villi of the intestinal wall is likely to interfere with triglyceride absorption from the intestinal contents, and may explain the decreased triglyceride concentrations when strongyle infection is present. If the infection persists, concentrations may increase as fat stores are mobilized. This finding in apparently healthy horses is interesting - but might not be a specific indicator, if the affected animals were ones with low body condition scores etc. None of the animals evaluated in this current study were obviously in a thin or starved condition, but as their clinical history was not known, it is not possible to know if they had suffered a period of weight loss/energy restriction, prior to euthanasia.

Cholesterol esters account for two-thirds of total cholesterol found in plasma. They act as storage and transport molecules which can be metabolized to release cholesterol and fatty acids. Cholesterol is used for membrane and lipoprotein formation, as well as hormone synthesis. Of the 3 cholesterol esters identified in the present study, the concentrations of cholesteryl ester and cholesteryl nitrolineate increased, whilst cholesteryl acetate was dramatically reduced (ratio 2.25) when infection was present (Table 1). A previous study also found increased concentrations of total cholesterol in strongyle infected donkeys [15]. This may be linked to the stress response initiated by the parasitic burden, leading to increased production and release of cholesterol based hormones such as epinephrine and corticosteroids.

Phosphoglycerides are the most abundant phospholipids in the majority of cell membranes [16]. In the present study, the concentrations of the majority of phosphoglycerides were increased with strongyle infection when compared with the control. A previous study proposed that parasitic nematodes produce and secrete glycoconjugates, in order to evade host immune responses [17]. In particular, phosphocholine, a small haptenic molecule frequently found on the antigens of parasites, is known to have immunomodulatory activities. Immunomodulation of the host's immune system allows persistence of the parasite and prevents the development of severe pathology, by inhibiting the host cellular immune responses [18].

Further validation of the findings from this study is required, to confirm that the observed alterations of the lipid profile were specifically due to the presence of strongyle infection, and is caused by a specific strongyle species. The level of significance chosen for this study suggests that the probability of these changes being due to chance alone was very low. However, there is a possibility that some alterations may not have been specific to strongylosis, and were instead linked to more general systemic responses such as inflammation. As these horses were being euthanased at a commercial abattoir for human consumption, they should have been healthy and generally free of overt systemic disease. For future development of this technique it may be advisable to concurrently measure, markers of inflammation and obtain a known clinical and management history of each animal. This would increase the probability that differences in lipid metabolite profile between horses with moderate- or high-intensity strongyle infections, compared with their uninfected counterparts are specific to strongyle infection and the pathology it causes.

A low number of ascarid eggs were found in two fecal samples from two different animals. Serum from one of these two horses was used for lipid extraction and analysis as a control animal, as this horse did not have a strongyle infection. It is possible that the ascarid infection could have interfered with results, affecting their reliability as an indicator of no strongyle infection. However, as the uninfected serum samples were pooled before HPLC-MS analysis, any effect of this minor ascarid infection noticed, should have been minimized by the presence of the five other ascarid-negative samples.

The high prevalence $(73.3 \%)$ of strongyle infection seen in the horses used for this study, is supported by previous publications [19, 20]. Because there is substantial variation in strongyle burden during the course of disease, future studies to evaluate the correlation between concentrations of metabolites and degree of strongyle infection should use quantitative techniques, such as the TaqMan PCR assay described by [21], to stratify the infection levels in the tested animals. The encysted immature non-laying strongyle stages are unlikely to contribute to the present finding, because no pathological lesions were detected in any of the control horses, ruling out any mucosal burden of cyathostomin parasites or encysted larval cyathostominosis (unpublished data).

In conclusion, this study has successfully used for the first time, high resolution HPLC-MS technique, to detect and tentatively identify lipids in equine serum on the basis of accurate mass. Statistical differences in 
Citation: Lamb J, Hallowell GD, Harris P, Zhu XQ, Elsheikha HM (2012) Lipidomic Analysis of Serum from Horses with Strongyle Infection. J Vet Sci Technol 3:122 doi:10.4172/2157-7579.1000122

the concentrations of 66 lipid metabolites between stronglye infected and uninfected horses were observed. These preliminary results demonstrate the potential use of this type of lipid analysis technique, for diagnosis of strongyle infection in equids. Future research in this area should be aimed at accurate and full identification of the lipid profiles of strongyle infected and healthy horses. Further studies to assess correlation between lipid concentrations and the intensity of strongyle infection, taking into account exact strongyle species in horses, is also required if development of a quantitative diagnostic test is to be achieved.

\section{Acknowledgements}

The authors are grateful for the funding received from WALTHAM. We also thank Prof. David Barrett and Dr. Catharine Ortori from the Centre for Analytical Bioscience, University of Nottingham for performing the serum analysis

\section{References}

1. Lichtenfels JR, Kharchenko VA, Dvojnos GM (2008) Illustrated identification keys to stongylid parasites (strongylidae: Nematoda) of horses, zebras and asses (Equidae). Vet Parasitol 156: 4-161.

2. Hodgkinson JE, Freeman KL, Lichtenfels JR, Palfreman S, Love S, et al. (2005) Identification of strongyle eggs from anthelmintic-treated horses using a PCRELISA based on intergenic DNA sequences. Parasitol Res 95: 287-292.

3. Traversa D, lorio R, Klei TR, Kharchenko VA, Gawor J, et al. (2007) New method for simultaneous species-specific identification of equine strongyles (nematoda, strongylida) by reverse line blot hybridization. J Clin Microbiol 45 : 2937-2942.

4. Nielsen MK, Peterson DS, Monrad J, Thamsborg SM, Olsen SN, et al. (2008) Detection and semi-quantification of Strongylus vulgaris DNA in equine faeces by real-time quantitative PCR. Int J Parasitol 38: 443-453.

5. Welti R, Mui E, Sparks A, Wernimont S, Isaac G, et al. (2007) Lipidomic analysis of Toxoplasma gondii reveals unusual polar lipids. Biochemistry 46: 13882-13890.

6. Zheng L, T'Kind R, Decuypere S, von Freyend SJ, Coombs GH, et al. (2010) Profiling of lipids in Leishmania donovani using hydrophilic interaction chromatography in combination with Fourier transform mass spectrometry. Rapid Commun Mass Spectrom 24: 2074-2082.

7. Nielsen MK, Vidyashankar AN, Andersen UV, Delisi K, Pilegaard K et al. (2010) Effects of fecal collection and storage factors on strongylid egg counts in horses. Vet Parasitol 167: 55-61.
8. Gordon HM, Whitlock HV (1939) A new technique for counting nematode eggs in sheep faeces. J Counc Sci Ind Res Aust 12: 50-52.

9. Bligh EG, Dyer WJ (1959)A rapid method of total lipid extraction and purification. Canadian Journal of Biochemistry and Physiology 37: 911-917.

10. Milhas D, Clarke CJ, Hannun YA (2010) Sphingomyelin metabolism at the plasma membrane: implications for bioactive sphingolipids. FEBS Lett 584 1887-1894.

11. Domingos MO, Barbaro KC, Tynan W, Penny J, Lewis DJ, et al. (2003) Influence of sphingomyelin and TNF-alpha release on lethality and local inflammatory reaction induced by Loxosceles gaucho spider venom in mice. Toxicon 42: 471-479

12. Pettus BJ, Chalfant CE, Hannun YA (2004) Sphingolipids in inflammation: roles and implications. Curr Mol Med 4: 405-418.

13. Moore BR, Abood SK, Hinchcliff KW (1994) Hyperlipemia in 9 miniature horses and miniature donkeys. J Vet Intern Med 8: 376-381.

14. Muñoz A, Riber C, Trigo P, Castejón F (2010) Hematology and clinical pathology data in chronically starved horses. J Equine Vet Sci 30: 581-589.

15. Parsani HR, Momin RR, Lateef A, Hemen D (2011) Haemato-biochemical alterations in helminths infected donkeys. WJAS 3: 112-114.

16. Boisseau P, Houdy P, Lahmani M (2010) Nanoscience: Nanobiotechnology and Nanobiology, Springer-Verlag, Berlin.

17. Jez, Joseph M (2007) Phosphatidylcholine Biosynthesis as a Potential Targe for Inhibition of Metabolism in Parasitic Nematodes. Curr Enzyme Inhib 3: 133 142

18. Grabitzki J, Lochnit G (2009) Immunomodulation by phosphocholinebiosynthesis, structures and immunological implications of parasitic PCepitopes. Mol Immunol 47: 149-163.

19. Gawor JJ (1995) The prevalence and abundance of internal parasites in working horses autopsied in Poland. Vet Parasitol 58: 99-108.

20. Von Samson-Himmelstjerna G, Traversa D, Demeler J, Rohn K, Milillo P, et al. (2009) Effects of worm control practices examined by a combined faecal egg count and questionnaire survey on horse farms in Germany, Italy and the UK. Parasit Vectors 2: S3.

21. Nielsen MK, Peterson DS, Monrad J, Thamsborg SM, Olsen SN, et al. (2008) Detection and semi-quantification of Strongylus vulgaris DNA in equine faeces by real-time quantitative PCR. Int J Parasitol 38: 443-453. 